\title{
ChemComm
}

\section{A thermally healable polyhedral oligomeric silsesquioxane (POSS) nanocomposite based on Diels-Alder chemistry ${ }^{\dagger}$}

Cite this: Chem. Commun., 2013,
49, 6755

Received 9th May 2013

Accepted 11th June 2013

DOI: $10.1039 / c 3 c c 43432 j$

www.rsc.org/chemcomm

A novel, tough, transparent, self-healing POSS nanocomposite material was prepared through a thermally reversible Diels-Alder (DA) reaction between furan-end functionalized POSS and bismaleimide.

Polymeric materials with self-healing ability to auto-repair physical damage have attracted great interest due to their increased safety index and service lifetime. They are particularly desirable for load-bearing applications where material failure repair is costly and has safety concerns. ${ }^{1-4}$ In engineering, cross-linked polymers are widely used because of their superior mechanical properties such as high modulus, high fracture strength, and solvent resistance. However, highly cross-linked polymeric materials are often brittle and apt to crack. Rendering them with self-healing ability would be an effective approach to repairing cracks. Two main approaches have been used to endow cross-linked polymeric materials with crack-healing ability: (1) incorporation of a healing agent, which is often in the form of microcapsules, into the matrix, ${ }^{1}$ and (2) using a special chemical bond, which can re-bond after being broken under certain conditions, to form the cross-linked network. In the first approach, capsules rupture upon being cracked and the release of a healing reagent from the capsules heals the crack. Although this method is simple and straightforward, it is not efficient for multiple self-healing. The second approach relies on the inherent reversibility of covalent bonds that can break and re-form upon exposure to certain environmental conditions. ${ }^{2}$ It is expected that the reversible bonds behave like conventional covalent bonds to constitute the cross-linked network under normal conditions, when crack happens the bonds break in the local area, the broken bonds can be re-built, and the process is repeatable for multiple repairing.

Diels-Alder (DA) reaction between an alkene and a diene is thermally reversible. Such a feature has been utilized to develop

Australian Future Fibres Research and Innovation Centre, Institute for Frontier Materials, Deakin University, Geelong, Vic 3216, Australia.

E-mail: tong.lin@deakin.edu.au

$\dagger$ Electronic supplementary information (ESI) available: Experimental details. See DOI: $10.1039 / \mathrm{c} 3 \mathrm{cc} 43432 \mathrm{j}$ amendable cross-linked networks. Wudl and co-workers ${ }^{2}$ reported a highly cross-linked network that can mend itself upon mild heating. Such a healable material was formed in its entirety by reversible formation of DA bonds between tri-functional maleimide and tetra-functional furan compounds. Liu and $\mathrm{Hsieh}^{5}$ prepared a thermally re-mendable epoxy resin through DA reactions between tri-functional maleimide and tri-functional furan monomers, which were both synthesized by using epoxy compounds as precursors. Thermally re-mendable epoxy resin has also been reported by Rong et $a l^{6}{ }^{6}$ and Palmese et al. ${ }^{7}$ In those works, apart from the use of DA bonds for intermonomer linkage, thermally stable bonds formed from the reaction between epoxide and anhydride groups were also used for network cross-linking.

Polyhedral oligomeric silsesquioxane (POSS) compounds are organic-inorganic hybrids with the empirical formula $\mathrm{R}_{n}\left(\mathrm{SiO}_{1.5}\right)_{n}$ $(n=8,10$, or 12$)$, and have dimensions comparable to most polymer segments or coils. The rigid and cubic cage can be considered as the smallest possible particles of silica. Each cage silicon atom is attached to a single $\mathrm{R}$ substituent, which can be reactive or nonreactive organic groups (e.g. glycidyl, phenyl, cyclohexyl), or organic-inorganic hybrids (e.g. $\left.-\mathrm{OSiMe}_{2} \mathrm{OPh}\right){ }^{8}$ The reactive organic groups offer a unique opportunity for preparing composite materials with the inorganic POSS core truly molecularly dispersed in the matrix. Compared to polymeric materials, the POSS nanocomposites usually have superior properties including higher use temperature, oxidation resistance and improved mechanical properties, as well as lower dielectric constant, flammability and heat evolution. ${ }^{9}$ However, to the best of our knowledge, POSS-based nanocomposites with self-healing ability have not been reported to date.

In this work, we report on a thermally mendable POSS nanocomposite prepared by directly cross-linking a POSS that brings eight furan-end functional groups with bismaleimide molecules via DA reaction. The POSS nanocomposite is stiff and transparent at room temperature, but can heal the cracks thermally and the healing has excellent repeatability.

Fig. 1 shows the reaction route to prepare the self-healing POSS nanocomposite. The synthesis details are provided in ESI. $\dagger$ FGPOSS was synthesized by reacting G-POSS with furfurylamine, and 

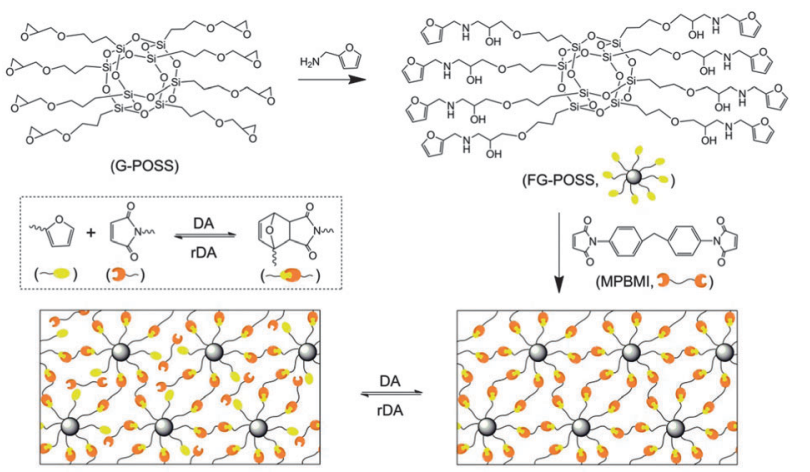

Fig. 1 Synthesis route to the self-healing POSS nanocomposite.

then was mixed with MPBMI in THF. After stirring for 3 hours, the THF was slowly removed by rotary evaporation. The resultant viscous liquid was poured into a mould and kept at room temperature for 20 hours, at $45{ }^{\circ} \mathrm{C}$ for 5 hours and at $95{ }^{\circ} \mathrm{C}$ for 18 hours to form a highly cross-linked nanocomposite. Fig. 2a shows the ${ }^{1} \mathrm{H}$ nuclear magnetic resonance (NMR) spectrum of FG-POSS. The resonance at $0.48-0.55 \mathrm{ppm}$ was assigned to the methylene protons $\left(-\mathrm{Si}-\mathrm{CH}_{2}-\right)$ of G-POSS whereas the peaks at 6.10-6.40 and $7.55 \mathrm{ppm}$ were ascribed to the resonance of the protons of furan rings. The characteristic peaks of $\mathrm{d}, \mathrm{e}, \mathrm{g}$ and $\mathrm{f}$ indicated that the epoxy ring group was opened and reacted with the amine group of furfurylamine. From the ratio of the integration intensity of $-\mathrm{Si}-\mathrm{CH}_{2}-$ protons of G-POSS and furan rings of furfurylamine, we estimated that the epoxy group reacted at a rate of $c a .98 \%$.

Fig. 2b shows the ${ }^{1} \mathrm{H}$ NMR results of in situ monitoring of the DA reaction between FG-POSS and MPBMI (see ESI $\dagger$ for a larger picture). An equivalent molar mixture of FG-POSS and MPBMI was dissolved in DMSO- $\mathrm{d}_{6}$ and the spectra were collected at room temperature immediately, after 2 and 24 hours, respectively. At the beginning, there were no obvious peaks at 5.1-5.3 ppm and 6.5-6.6 ppm, which corresponded to the characteristic $\mathrm{H}_{\mathrm{h}^{\prime}}, \mathrm{H}_{\mathrm{i}^{\prime}}$ and $\mathrm{H}_{\mathrm{j}^{\prime}}$ peaks of the DA adduct. However, these characteristic
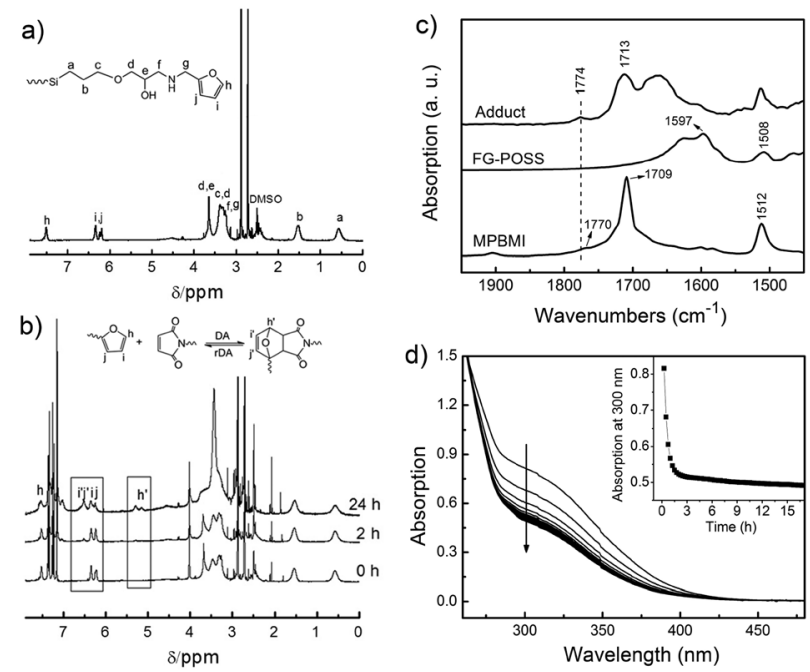

Fig. 2 (a) ${ }^{1} \mathrm{H}$ NMR spectrum of FG-POSS. (b) Time-dependent ${ }^{1} \mathrm{H}$ NMR spectra of the adduct of FG-POSS and MPBMI. (c) FTIR spectra of FG-POSS, MPBMI and the adduct. (d) Time-dependent UV spectra of the adduct of FG-POSS and MPBMI. The inset of (d) shows the decrease of the absorption at $300 \mathrm{~nm}$ with time. peaks appeared after 2 hours (the rectangular area highlighted in Fig. 2b). After 24 hours, the characteristic $\mathrm{H}_{\mathrm{h}^{\prime}}$ peak (5.1, 5.3 ppm) became more evident, and the intensity of $\mathrm{H}_{i}$ and $\mathrm{H}_{\mathrm{j}}$ peaks (6.3 and $6.2 \mathrm{ppm}$ ) decreased, while the intensity of $\mathrm{H}_{\mathrm{i}^{\prime}}$ and $\mathrm{H}_{\mathrm{j}^{\prime}}$ peaks (6.6 and $6.5 \mathrm{ppm}$ ) increased, thus confirming the DA reaction between FG-POSS and MPBMI.

The DA reaction was also verified by Fourier transform infrared (FTIR) and UV-visible spectroscopy. Fig. 2c shows the FTIR spectra of MPBMI, FG-POSS, and the DA adduct. The spectrum of MPBMI showed the benzene framework absorption band at $1512 \mathrm{~cm}^{-1}$, the carbonyl doublet characteristic peak at $1770 \mathrm{~cm}^{-1}$, and the $\mathrm{C}=\mathrm{O}$ stretching vibrations of the imide group at $1709 \mathrm{~cm}^{-1} \cdot{ }^{10}$ In the spectrum of FG-POSS, the absorption bands at 1508 and $1597 \mathrm{~cm}^{-1}$ were ascribed to the furan heterocycle. ${ }^{11}$ The FTIR spectrum of the DA adduct showed a shift of the carbonyl doublet characteristic peak and the imide $\mathrm{C}=\mathrm{O}$ stretching vibrations to higher wavenumbers of 1774 and $1713 \mathrm{~cm}^{-1}$, respectively, which indicates the formation of DA bonds between furan and maleimide groups.

Fig. $2 \mathrm{~d}$ shows the UV-visible spectra recorded during the DA reaction. The decline of the absorption at $c a .300 \mathrm{~nm}$ is due to the conversion of maleimide to the DA adduct (loss of the $\mathrm{O}=\mathrm{C}-$ $\mathrm{C}=\mathrm{C}-\mathrm{C}=\mathrm{O}$ conjugation $).{ }^{12}$ At the first three hours, the absorption at ca. $300 \mathrm{~nm}$ decreased quickly and nearly $90 \%$ of maleimide groups were consumed. After three hours, the absorption just changed slightly, indicating that the reaction between FGPOSS and MPBMI proceeded very quickly at room temperature.

The as-prepared POSS nanocomposite looks transparent (Fig. 3a). Without cross-linking reagents, opaque whitish films are usually formed from a single POSS compound because of its high symmetry and crystallinity. ${ }^{13}$ The formation of a transparent product verified that the dispersion of FG-POSS was at the molecular level which typically resulted from covalent bonding. The POSS nanocomposite has a compressive modulus of $1.0 \mathrm{GPa}$ and a yield strength of $118 \mathrm{MPa}$ (Fig. 3a), which is comparable to that of commercial cross-linked epoxy resins (102 to $170 \mathrm{MPa}) .{ }^{14}$

To prove the healing ability, the POSS nanocomposite was deliberately cracked, and the damage was thermally mended at $135{ }^{\circ} \mathrm{C}$ and $150{ }^{\circ} \mathrm{C}$, respectively. The cracked sample in Fig. $3 \mathrm{~b}$ was heated at $135{ }^{\circ} \mathrm{C}$ for 30 minutes and then cooled down naturally in the oven. After thermal treatment, the cracks were successfully healed without any scar left. Fig. 3c shows the
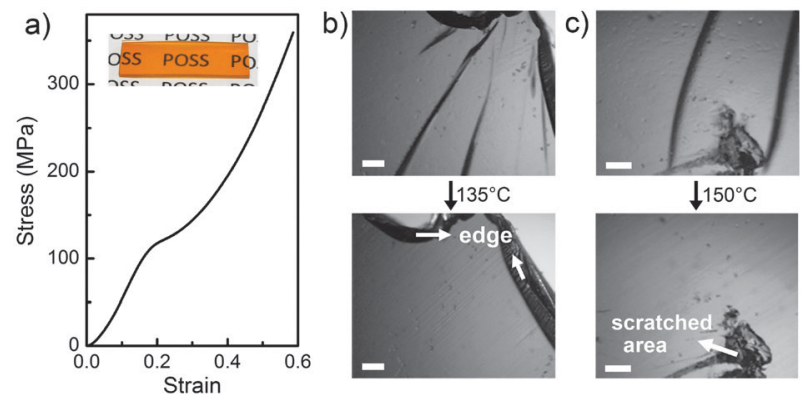

Fig. 3 (a) Compressive stress-strain curve and appearance of the as-prepared POSS nanocomposite. (b, c) Visual inspection of the crack-healing behaviour: cracked samples were treated in the oven at (b) $135^{\circ} \mathrm{C}$ and (c) $150{ }^{\circ} \mathrm{C}$ for $30 \mathrm{~min}$, respectively, and then naturally cooled down in the oven. Scale bar: $1 \mathrm{~mm}$. 
cross-linked POSS nanocomposite with the surface heavily scratched. After being heated at $150{ }^{\circ} \mathrm{C}$ for 30 minutes, the cracks were healed but the scratched surface remained unhealed due to the material loss during scratching. These results suggest that healing takes place only when the damaged parts are closely contacted during heat treatment.

Differential scanning calorimetry (DSC) was used to study the thermally reversible reaction in the cross-linked POSS nanocomposite. Fig. 4a shows the heating curves of the DSC analysis. In the first heating curve, there was a broad endothermic peak from $94{ }^{\circ} \mathrm{C}$ to $210{ }^{\circ} \mathrm{C}$. After the sample was cooled down to $20{ }^{\circ} \mathrm{C}$, the second heating was conducted. In the second heating curve, however, no endothermic peak was observed. Thus, it is reasonable to deduce that the endotherm appearing in the first heating curve results from the retro-DA reaction. ${ }^{6}$ Because the time of the cooling process is not enough for the disconnected furan and maleimide moieties to reconnect, no retro-DA reaction occurred in the second heating scan. Here it should be pointed out that although the retro-DA reaction happens in a wide temperature range from $94{ }^{\circ} \mathrm{C}$ to $210{ }^{\circ} \mathrm{C}$ as indicated in the DSC curve, the temperature for self-healing treatment should not be higher than $180{ }^{\circ} \mathrm{C}$ because of the polymerization of free maleimide moieties at an elevated temperature. ${ }^{6}$ This reaction is unfavourable to the thermal reversibility of the DA reaction because it converts maleimide to irreversible bonds.

DSC was also used to study the retro-DA reaction in the crosslinked POSS nanocomposite during the thermal healing process. The sample was heated from room temperature to $135^{\circ} \mathrm{C}$, kept at that temperature for 30 minutes, and then cooled down to room temperature naturally. This process was repeated until the fifth heating, where the sample was heated to $240{ }^{\circ} \mathrm{C}$. Fig. 4 b shows the heating curves of the DSC analysis. Compared to the first heating scan, the amount of heat flow in the second heating scan significantly decreased. In the following heating scans, the amount of heat flow further decreased slightly. This decrease in the amount of heat flow implies that the retro-DA reaction became more difficult with heating cycles. ${ }^{6}$ The increased difficulty in the
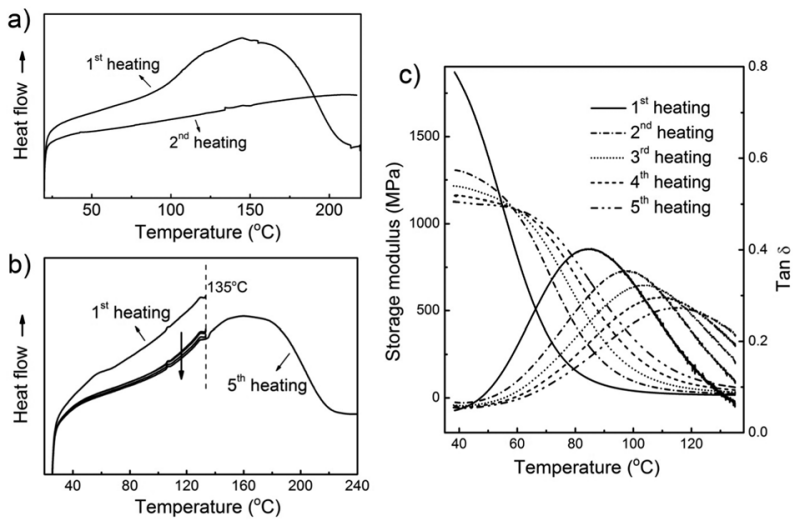

Fig. 4 ( $a, b)$ DSC heating curves of the cross-linked POSS nanocomposite: (a) sample was first heated to $220^{\circ} \mathrm{C}$, and then cooled down to $20^{\circ} \mathrm{C}$ in the DSC cell, followed by the second heating; (b) the sample was first heated to $135^{\circ} \mathrm{C}$, kept at that temperature for $30 \mathrm{~min}$, and then cooled down to $25^{\circ} \mathrm{C}$, followed by the second heating. This process was repeated until the fifth heating, where the sample was heated to $240{ }^{\circ} \mathrm{C}$. (c) Storage modulus and loss factor $\tan \delta$ of the cross-linked POSS nanocomposite at different healing cycles.
retro-DA reaction was also observed in the following dynamic mechanical analysis (DMA) measurement. Here, it should be noted that although the amount of heat flow decreased gradually with heating, an obvious endothermic peak was observed during the fifth heating scan, which implies the good thermal reversibility of the DA reaction even after repeated heating.

The dynamic mechanical properties of the cross-linked POSS nanocomposite were studied by DMA. The sample was tested with the repeated heating and cooling process five times. Fig. 4c shows the time dependence of the storage modulus and the loss factor tan $\delta$ of the POSS nanocomposite. In the first heating scan, even though the storage modulus at $38{ }^{\circ} \mathrm{C}$ was as high as $1870 \mathrm{MPa}$, it started dropping at that temperature, which indicates that the sample started softening. The time dependence of $\tan \delta$ shows a peak at $85{ }^{\circ} \mathrm{C}$. In the second heating scan, the storage modulus at $38{ }^{\circ} \mathrm{C}$ decreased to $1310 \mathrm{MPa}$. This decrease was caused by the retro-DA reaction during the first heating cycle. Some of the disconnected furan and maleimide moieties did not get enough time to reconnect, thus reducing the stiffness of the material. In the following heating cycles, the storage modulus at $38{ }^{\circ} \mathrm{C}$ further decreased slightly, but still remained as high as $1120 \mathrm{MPa}$ in the fifth heating scan. In addition, the rate of the decrease in storage modulus decreased with the heating cycles, which implies that the retro-DA reaction became more difficult with heating. ${ }^{6}$ This phenomenon was also evidenced by the increase in the $\tan \delta$ peak value with the heating cycles. The tan $\delta$ peak value increased from $85{ }^{\circ} \mathrm{C}$ for the original sample to $116{ }^{\circ} \mathrm{C}$ for the sample in the fifth heating scan.

In conclusion, a novel transparent, self-healing POSS nanocomposite has been prepared successfully through a thermally reversible DA reaction between furan-end functionalized POSS and bismaleimide. The material is tough and solid at room temperature. Upon being cracked, the cracked parts can be healed by heating the material to cleave the DA bonds at above $94{ }^{\circ} \mathrm{C}$ and then reconnecting the furan and maleimide moieties at a lower temperature. This process is reversible and can be used to mend the internal cracks of the material.

\section{Notes and references}

1 S. R. White, N. Sottos, P. Geubelle, J. Moore, M. R. Kessler, S. Sriram, E. Brown and S. Viswanathan, Nature, 2001, 409, 794.

2 X. Chen, M. A. Dam, K. Ono, A. Mal, H. Shen, S. R. Nutt, K. Sheran and F. Wudl, Science, 2002, 295, 1698.

3 R. P. Wool, Soft Matter, 2008, 4, 400.

4 S. Burattini, B. W. Greenland, D. Chappell, H. M. Colquhoun and W. Hayes, Chem. Soc. Rev., 2010, 39, 1973.

5 Y. L. Liu and C. Y. Hsieh, J. Polym. Sci., Part A: Polym. Chem., 2006, 44, 905.

6 Q. Tian, Y. C. Yuan, M. Z. Rong and M. Q. Zhang, J. Mater. Chem., 2009, 19, 1289.

7 A. M. Peterson, R. E. Jensen and G. R. Palmese, ACS Appl. Mater. Interfaces, 2010, 2, 1141.

8 K. Liang, G. Li, H. Toghiani, J. H. Koo and C. U. Pittman, Chem. Mater., 2006, 18, 301.

9 G. Z. Li, L. Wang, H. Toghiani, T. L. Daulton, K. Koyama and C. U. Pittman, Macromolecules, 2001, 34, 8686.

10 M. Sava, J. Appl. Polym. Sci., 2009, 112, 1399.

11 C. Vilela, L. Cruciani, A. J. Silvestre and A. Gandini, RSC Adv., 2012, 2, 2966.

12 A. Gandini, D. Coelho, M. Gomes, B. Reis and A. Silvestre, J. Mater. Chem., 2009, 19, 8656.

13 H. Araki and K. Naka, Macromolecules, 2011, 44, 6039.

14 S. H. Goodman, Handbook of thermoset plastics, Noyes Publications, Westwood, New Jersey, USA, 2nd edn, 1998. 